13. Loss J, Eichhorn C, Reisig V et al (2007) Qualitätsmanagement in der Gesundheitsförderung. Prävention und Gesundheitsförderung 2:199-206

14. Mayring P (2000) Qualitative Inhaltsanalyse. Grundlagen und Techniken, 7. Aufl. Deutscher Studien Verlag, Weinheim

15. Meltzer LS, Huckabay LM (2004) Critical care nurses' perceptions of futile care and its effect on burnout. Am J Crit Care 13:202-208

16. Mitchell PH, Lang NM (2004) Framing the problem of measuring and improving healthcare quality: has the quality health outcomes model been useful? Med Care 42:I14-II11

17. Mobley MJ, Rady MY, Verheijde JL et al (2007) The relationship between moral distress and perception of futile care in the critical care unit. Intensive Crit Care Nurs 23:256-263

18. Papathanassoglou ED, Karanikola MN, Kalafati M et al (2012) Collaboration with physicians, and moral distress among European intensive care nurses. Am J Crit Care 21:e41-e52

19. Pauly BM, Varoe C, Storch J (2012) Framing the issues: moral distress in health care. HEC Forum 24:1-11

20. Piers RD, Van den Eynde M, Steeman E et al (2012) End-of-life care of the geriatric patient and nurses' moral distress. J Am Med Dir Assoc 13:e7-e80

21. Piers RD, Azoulay E, Ricou B et al (2011) Perceptions of appropriateness of care among European and Israeli intensive care unit nurses and physicians. JAMA 306:2694-2703

22. Puntillo KA, McAdam JL (2006) Communication between physicians and nurses as a target for improving end-of-life care in the intensive care unit: challenges and opportunities for moving forward. Crit Care Med 34:332-340

23. Puntillo KA, Benner P, Drought T et al (2001) Endof-life issues in intensive care units: a national random survey of nurses' knowledge and beliefs. Am J Crit Care 10:216-229

24. Raines ML (2000) Ethical decision making in nurses relationships among moral reasoning coping style, and ethics stress. JONAS Healthc Law Ethics Regul 2(1):29-41

25. Reiter-Theil S, Mertz M, Albisser Schleger H et al (2011) Klinische Ethik als Partnerschaft - oder wie eine ethische Leitlinie für den patientengerechten Einsatz von Ressourcen entwickelt und implementiert werden kann. Ethik Med 23:93-105

26. Reiter-Theil S, Mertz M, Schürmann J et al (2011) Evidence - competence - discourse: the theoretical framework of the multi-centre clinical ethics support project METAP. Bioethics 25:403-412

27. Rice EM, Rady MY, Hamrick A et al (2008) Determinants of moral distress in medical and surgical nurses at an adult acute tertiary care hospital. $J$ Nurs Manag 16:360-373

28. Schwenzer KJ, Wang L (2006) Assessing moral distress in respiratory care practitioners. Crit Care Med 34:2967-2973

29. Dam S van der, Schols JM, Kardol TJ et al (2013) The discovery of deliberation. From ambiguity to appreciation through the learning process of doing Moral Case Deliberation in Dutch elderly care. Soc Sci Med 83:125-132

30. Wilkinson JM (1988) Moral distress in nursing practice: experience and effect. Nurs Forum 23:16-29

Med Klin Intensivmed Notfmed 2014 .

109:354-363

DOI 10.1007/s00063-014-0387-7

Online publiziert: 29. Mai 2014

๑) Springer-Verlag Berlin Heidelberg 2014

\author{
N. Verma ${ }^{1} \cdot$ P. Willeke ${ }^{1} \cdot$ P. Bicsán ${ }^{2} \cdot$ P. Lebiedz ${ }^{3} \cdot$ H. Pavenstädt ${ }^{1}$ P. Kümpers ${ }^{1}$ \\ ${ }^{1}$ Medizinische Klinik D, Abteilung für Allg. Innere Medizin, Nephrologie \\ und Rheumatologie, Universitätsklinikum Münster \\ ${ }^{2}$ Geschäftsbereich IT, Universitätsklinikum Münster \\ ${ }^{3}$ Department für Kardiologie und Angiologie, Universitätsklinikum Münster
}

\title{
Erratum zu: Altersadjustierte D-Dimer-Grenzwerte in der Diagnostik thrombembolischer Ereignisse
}

\section{Validierung in der Notaufnahme}

Unter dem Punkt „Einhaltung der ethischen Richtlinien“" wurde vom Verlag eine falsche Formulierung abgedruckt. Wie im Abschnitt „Patienten und Studiendesign“ erwähnt, hat die verantwortliche Ethikkommission die nachträgliche Einholung der Patienteneinwilligungen für nicht notwendig erachtet.

Wir bitten, die korrekte Angabe zu beachten und den Fehler $\mathrm{zu}$ entschuldigen.

Die Redaktion

\section{Korrespondenzadresse}

\section{PD Dr. P. Kümpers}

Medizinische Klinik D,

Abteilung für Allg. Innere Medizin, Nephrologie und Rheumatologie, Universitätsklinikum Münster Albert-Schweitzer-Campus 1 , 48149 Münster philipp.kuempers@ukmuenster.de
Die Online-Version des Originalartikels können Sie unter http://dx.doi.org/ 10.1007/s00063-013-0265-8 finden. 